\title{
Organisation et contrôle de qualité dans la surveillance biologique et l'exposition professionnelle aux métaux
}

\author{
Quality control in biological monitoring \\ and occupational exposure to metals
}

\section{Alain PINEAU*(1), Olivier GUILLARD ${ }^{(2)}$}

(1) Laboratoire de Toxicologie-Hygiène-Sécurité, Faculté de Pharmacie de Nantes

(2) Laboratoire de Biochimie et de Toxicologie, Faculté de Médecine de Poitiers

* Auteur à qui adresser la correspondance : Prof. Alain PINEAU, Laboratoire de Toxicologie-Hygiène-Sécurité, Faculté de Pharmacie, 1, rue Gaston Veil - BP 53508 - 44035 NANTES Cedex 1 Tél : (33) 240412855 - Fax : (33) 240412889 - E-mail : Alain.Pineau@ sante.univ-nantes.fr

(Reçu le 10 mai 2001 ; accepté le 20 juin 2001)

\section{RÉSUMÉ}

En France, actuellement, le contrôle qualité pour la surveillance biologique de l'exposition professionnelle aux métaux se limite à la plombémie. Après une évocation de la législation relative à la détermination du plomb dans le sang, le texte aborde l'organisation du contrôle qualité plombémie de la préparation des échantillons à l'analyse des résultats. La présentation d'un aspect pratique de ce contrôle qualité (modalité d'inscription) a pour but d'aider les laboratoires à s'y inscrire. En conclusion, dans une démarche qualité, si la participation à ce contrôle national plombémie est obligatoire pour obtenir l'agrément, elle n'est pas suffisante car elle doit être associée également à la participation à des contrôles internationaux et à l'utilisation de contrôles commerciaux.

\section{MOTS-CLÉS}

Contrôle qualité, sang, plomb, exposition professionnelle.

\section{SUMMARY}

Quality control in France for biological monitoring of occupational exposure to metals is currently limited to the determination of plasma lead levels. After a brief consideration of the legislation involved, the present report describes the organisation of this quality control procedure from the preparation of samples to the analysis of results. The presentation of a practical aspect (registration requirements) is intended to assist laboratoires in qualifying for official approval. Although participation in this national quality control procedure for plasma lead levels is required to obtain approval, it is in fact inadequate and should be associated withparticipation in international controls and the use of commerical controls.

\section{KEY-WORDS}

Quality Control, blood, lead, occupational exposure. 


\section{Introduction}

En France, la législation relative au contrôle qualité dans la surveillance biologique de l'exposition professionnelle aux métaux se limite à la plombémie. La détermination des autres métaux n'est pas soumise à une obligation de contrôle qualité.

Cependant, comme pour tout dosage biologique, on doit encourager les laboratoires qui effectuent des dosages de minéraux dans le sang ou les urines, à participer à des contrôles de qualité externes ou à intégrer dans les séries analytiques des échantillons commerciaux dont les concentrations sont relativement ciblées $(1,2)$.

Dans cette présentation, nous nous limiterons volontairement au plomb.

\section{Surveillance de l'exposition au plomb - Législation}

Pour surveiller l'exposition professionnelle au plomb, on utilise souvent le sang pour déterminer le niveau d'imprégnation des individus. Compte tenu de l'utilisation des produits à base de plomb et de leur impact sur la santé, n'oublions pas que les maladies causées par le plomb et ses composés constituent le tableau 1 des maladies professionnelles; il est donc essentiel que les résultats analytiques soient fiables. En effet, les résultats peuvent avoir des répercussions sur l'employé, en l'écartant de son poste de travail, et sur l'employeur en l'obligeant à prendre des mesures d'hygiène. Le résultat de la plombémie doit donc être fiable.

C'est pourquoi, dès 1988, le ministère des affaires sociales et de l'emploi, en collaboration avec les industriels et les salariés, a déterminé une procédure de contrôle de l'exposition au plomb (3). L'article $4 \mathrm{du}$ titre 2 du décret 88-120 mentionne qu'il faut effectuer "un dosage de la plombémie de chaque travailleur exposé" ; des valeurs seuils sont indiquées et surtout ces contrôles "doivent être pratiqués par un organisme agréé par arrêté des ministres, chargés du travail et de l'agriculture".

L'arrêté du 15 septembre 1988 (4) fixe les modalités d'application du décret du 1er février (3). En ce qui concerne les indicateurs de surveillance médicale, cet arrêté précise que "la plombémie est l'examen le plus approprié pour juger de la contamination d'un sujet", en attirant "l'attention sur la rigueur nécessaire pour que cet examen soit fiable" en insistant sur le "choix du laboratoire garantissant la validité de ses résultats, notamment par la pratique de contrôles interlaboratoires". Dans ce même texte, on trouve également des "valeurs de référence" ou "indices significatifs spécifiques". La plombémie y est exprimée en $\mu \mathrm{g} / 100 \mathrm{ml}$.

L'arrêté du 11 avril 1988 (5) précise dans l'annexe II, que la méthode d'analyse de la plombémie est la "spectroscopie d'absorption atomique". Ce texte est complété par l'arrêté du 14 novembre 1996 (6) relatif au dossier que doit remplir un laboratoire qui sollicite un agrément pour mesurer la plombémie. Ce dossier doit mentionner les équipements disponibles, la qualification du personnel, les tarifs pratiqués, tout élément propre à attester de l'expérience et la compétence en matière de plombémie, l'engagement à suivre les méthodes analytiques de référence et à se soumettre à tout contrôle ou évaluation externe.

Ce texte est essentiel car il constitue un précurseur de l'assurance qualité en biologie appliquée au milieu professionnel en imposant à la fois une technique analytique et la participation à des validations externes. De plus, le texte précise que l'agrément, accordé pour une durée déterminée (en pratique 1 ou 3 ans) est révocable et que le laboratoire agréé doit adresser chaque année au Ministère du Travail un bilan d'activité.

La législation européenne s'appuie surtout sur la directive 82/605/CEE (7). Dans le texte, nous retrouvons les mentions de plombémie, de valeurs de référence, de méthodologie par "spectroscopie d'absorption atomique" et de "programmes de contrôle de qualité appropriés".

\section{Organisation du contrôle de qualité plombémie}

Le contrôle de qualité plombémie est organisé par l'Agence du Médicament à la demande du Ministère du Travail depuis novembre 1992. Dans un premier temps, la seule participation à ce contrôle permettait d'obtenir l'agrément. Rapidement, le Ministère a, en plus de cette participation, exigé une qualité de résultats, afin de garantir la fiabilité des laboratoires. Depuis l'année 2000, c'est l'Agence Française de Sécurité Sanitaire des Produits de Santé (AFSSAPS) - Unité Contrôle National de Qualité qui organise ce contrôle de qualité. Quatre opérations de contrôle sont organisées chaque année (8). En moyenne, 65 laboratoire d'établissements publics ou de laboratoires d'analyses de biologie médicale y participent en étant identifiés par un numéro anonyme, dont seule l'AFSSAPS connaît la correspondance. Chaque contrôle (3 concentrations différentes) nécessite l'inclusion de prélèvements sanguins de deux patients groupes Rh identiques et VIH, VHB et VHC négatifs. Après homogénéisation, le pool est distribué en trois lots. Deux lots seront surchargés puis, après 
homogénéisation, analysés, répartis en petits tubes Sarstedt, codifiés et distribués. Afin de tester la reproductibilité, au cours de l'année, quelques échantillons (3 en 1999) sont envoyés en double. Les échantillons sont acheminés par la poste. Afin d'étudier la stabilité des échantillons, ceux-ci sont conservés à $+4{ }^{\circ} \mathrm{C}$ et à température ambiante puis analysés pendant 4 semaines sur le site de préparation des contrôles. Les laboratoires doivent rendre leurs résultats dans un délai maximum de deux semaines.

\section{Analyse des résultats $\mathrm{du}$ Contrôle National Plombémies (8-9)}

Après chaque contrôle, les laboratoires reçoivent une feuille de leur bilan indiquant les numéros d'échantillons, les moyennes, leurs résultats ; à la fin de l'année, il reçoivent un bilan chiffré général. Les principes des calculs reposent sur la notion de seuil d'acceptabilité ou d'écart à la valeur vraie. Les limites de tolérance sont de $3,0 \mu \mathrm{g} / 100 \mathrm{ml}$ de sang pour une plombémie de $10 \mu \mathrm{g} / 100 \mathrm{ml}$ et de $5,0 \mu \mathrm{g} / 100 \mathrm{ml}$ pour une plombémie de $60 \mu \mathrm{g} / 100 \mathrm{ml}$. Le détail des calculs a été expliqué dans les Annales du Contrôle National de Qualité (10) et dans un numéro spécial de la revue de l'Institut Supérieur de Santé (11) qui présentait tous les contrôles européens. La synthèse annuelle a pour but d'évaluer la performance du participant en indiquant pour chaque échantillon la comparaison à la moyenne (notée sur 100), la récupération des ajouts (notée sur $100)$ et pour certains échantillons la reproductibilité (notée sur 20).

La synthèse des résultats 1999 (9) met en évidence un bon pourcentage de participation $(>83 \%$ ), avec la spectrométrie d'absorption atomique électrothermique comme la technique analytique la plus utilisée $(>90 \%)$.

La participation régulière à ce contrôle a entraîné une très nette amélioration de la qualité des résultats. Ainsi nous observons, pour des concentrations identiques, une diminution de la dispersion (CV \%) entre 1994 et $1999(25,61 \%$ pour $10,53 \mu \mathrm{g} / 100 \mathrm{ml}$ en 1994 vs 10,9 $\%$ pour 10,08 en 1999 de $14,47 \%$ pour $44,81 \mu \mathrm{g} / 100$ $\mathrm{ml}$ vs $10,4 \%$ pour 44,22 ). Parallèlement, on note une élévation très significative de la moyenne sur 200 qui a évolué de 103 en 1994 à 158 en 1999 résultant d'une augmentation des notes des écarts à la moyenne (83/100 vs 55) et de la récupération des ajouts $(75 / 100$ vs 48 ). En ce qui concerne la reproductibilité, les résultats sont globalement satisfaisants avec une note moyenne des participants de 15/20.

\section{Contrôle de Qualité Plombémie - Aspect pra- tique}

Pour demander un agrément, il faut, avant fin octobre, adresser un dossier complet conformément à l'arrêté du 14 novembre 1999 (6) au Ministère de l'Emploi et de la Solidarité - Direction des Relations du Travail - Bureau CT4, Plombémie - 20 bis rue d'Estrées - 75700 PARIS 07 SP.

L'agrément est conditionné à des compétences et à des moyens. Il est très utile de participer à des contrôles de qualité externe (1-2) et il est indispensable d'avoir déjà une participation au circuit national plombémie organisé par l'AFSSAPS (Unité Contrôle National de Qualité - Plombémie - 143/147 Boulevard Anatole France 93285 - Saint-Denis cedex).

Il faut noter la différence d'utilisation des résultats des contrôles qualité. Pour l'AFSSAPS, ils permettent d'aider les laboratoires à se positionner et éventuellement à s'améliorer. Pour le Ministère du Travail, ils sont employés comme élément d'appréciation de la compétence dans l'objectif de la délivrance de l'agrément.

\section{Conclusion}

En France, légalement, le contrôle de qualité dans la surveillance biologique de l'exposition professionnelle aux métaux se limite au plomb et donc à la détermination de la plombémie. L'AFSSAPS organise un contrôle qualité depuis 1992. Le Contrôle National de Qualité Plombémie est comparable dans sa réalisation, ses modes de calcul et ses résultats aux autres contrôles internationaux. La participation au contrôle national est obligatoire mais pas suffisante pour obtenir l'agrément du Ministère du Travail. En effet, celui-ci est donné en fonction de la régularité de participation aux contrôles et de la qualité des résultats (plus de $50 \%$ de réponses correctes) mais également en fonction de la participation à des contrôles de qualité internationaux. Cette démarche exigeante apporte une sécurité supplémentaire dans les résultats fournis par les laboratoires.

Les contrôles de qualité sont indispensables, mais leur fréquence relativement faible (au mieux un par mois, plus fréquemment un par trimestre) doit orienter les laboratoires qui dosent le plomb à inclure dans leurs séries analytiques des échantillons de contrôles internes commercialisés et si possible certifiés $(1,2,8)$.

Dans les années à venir, il serait souhaitable que la démarche "contrôle qualité" s'étende à d'autres minéraux dans les milieux biologiques comme c'est le cas dans la plupart des pays anglo-saxons et scandinaves. 


\section{Références}

1. Pineau A., Guillard O., Baruthio F., Chappuis P. Contrôles de qualité interne et externe. In : Baruthio F. Assurance de qualité de l'analyse des éléments-trace en biologie. Tec et Doc Lavoisier, Éditions Médicales Internationales, $1997 ; 183-202$.

2. Pineau A., Guillard O. Généralités ; In : Guillard O. et Pineau A. Techniques d'analyse des oligoéléments chez l'homme, volume 2. Tec et Doc Lavoisier, Éditions Médicales Internationales, 2001 (à paraître), 1-14.

3. Décret $88-120$ du $1^{\text {cr }}$ février 1988 relatif à la protection des travailleurs exposés au plomb métallique et à ses composés. J.O. 5 février 1988, 1758-1760.

4. Arrêté du 15 septembre 1988 portant application de l'article 16 du décret $n^{\circ} 88-120$ du ler février 1988 et fixant les instructions techniques que doivent respecter les médecins du travail assurant la surveillance médicale des travailleurs exposés au plomb métallique et à ses composés et les valeurs de référence des paramètres biologiques représentatifs de l'exposition de ces travailleurs à ce toxique. J.O. 22 octobre 1988, 13 346-13 350.

5. Arrêté du 11 avril 1988 relatif au contrôle de l'exposition des travailleurs au plomb métallique et à ses composés. J.O. 19 avril 1988, 5188.
6. Arrêté du 14 novembre 1990 relatif au contrôle de l'exposition des travailleurs au plomb métallique et à ses composés. J.O., 23 novembre 1990, 14418.

7. Directive du Conseil du 28 juillet 1982 concernant la protection des travailleurs contre les risques liés à une exposition au plomb métallique et à ses composés ioniques pendant le travail - 82/605/CEE. JOCE. 23 août 1982, $\mathrm{N}^{\circ}$ L 247/12, 197-206.

8. Guillard O., Pineau A. Contrôle National de Qualité Plombémie 1998. Annales du Contrôle National de Qualité, 2000, n² 21, 139-148.

9. Guillard O., Pineau A. Contrôle National de Qualité Plombémie 1999. Annales du Contrôle National de Qualité, 2001, à paraître.

10. Guillard O., Pineau A. Contrôle National de Qualité Plombémie 1996. Annales du Contrôle National de Qualité, 1997, n 10, 71-78.

11. Guillard O., Pineau A., Piriou A. French external quality assessment schemes for lead in blood and aluminium in plasma and dialysis water. Ann. Ist. Super. Sanitá, 1996, 32, 241-245. 